\title{
Spot-like Proton Acceleration in SNR Shocks: Implications for the Energy Spectra
}

\section{Tatyana Liseykina*}

Rostock University, Germany

E-mail: tatyana.liseykina@uni-rostock.de

\section{Adrian Hanusch}

Rostock University, Germany

E-mail: adrian.hanusch@uni-rostock.de

\section{Mikhail Malkov}

University of California, San Diego, USA

E-mail: mmalkov@ucsd.edu

\section{Felix Aharonian}

Dublin Institute for Advanced Studies, Dublin, Ireland Max-Planck-Institut für Kernphysik, Heidelberg, Germany

E-mail: felix.aharonian@mpi-hd.mpg.de

\begin{abstract}
Cosmic ray (CR) spectra, both measured upon their arrival at the Earth atmosphere and inferred from the emission in supernova remnants (SNR), appear to be significantly steeper than the "standard" diffusive shock acceleration (DSA) theory predicts. Although the CR spectra steepen due to propagation losses, this steepening falls short to explain the data. We investigate whether the variable acceleration conditions on the SNR shock suffice to produce the necessary extra steepening. To this end, we perform two-dimensional hybrid simulations of a shock propagating into a SNR shock environment where the magnetic field inclination to the shock front varies. The rationale behind this approach is the strong dependence of the DSA efficiency upon the field angle. We find that the variation of shock obliquity along its face results in a noticeable steepening of the DSA spectrum: as compared to simulations of quasi-parallel shocks, we observe an increase of the spectral index by $\Delta q=0.1-0.15$.
\end{abstract}

36th International Cosmic Ray Conference -ICRC2019-

July 24th - August 1st, 2019

Madison, WI, U.S.A.

${ }^{*}$ Speaker. 


\section{Introduction}

The high precision measurements of cosmic ray (CR) spectra available nowadays reveal noticeable disagreements with the theoretical predictions. Many of those are build upon the theory of diffusive shock acceleration (DSA), which is able to explain the production of high-energy particles in a variety of shocks (see, e.g., [1] and references therein). The "standard" DSA theory is known to be insensitive to most of the shock parameters and predicts for the accelerated particles a power-law spectrum in momentum, $f \propto p^{-q}$, with the index, $q=3 r /(r-1)$, which depends only on the shock compression $r$. Some difference between predicted and observed spectra is expected because of factors not determined by the experiments, primarily propagation losses, whose scaling in energy is not known precisely. However, there are two types of observations where propagation losses can be taken out of consideration when testing the DSA predictions. The first type is pertinent to the spectrum of the ratio of two different elements attributable to the same or a similar accelerator. At least in one such case, the theory has already faced a serious challenge when a $\Delta q \approx 0.1$ difference between the proton and helium rigidity spectra was firmly established $[2,3,4]$. Explanations have been given (see [5, 6] for a review), but only a few of them address the proton spectrum steepening relative to helium at the DSA level (see [7] and the references therein). These explanations do not vindicate the DSA ultimately because of the second type of disagreements associated with observations of individual particle spectra in SNRs. The spectra are measured in situ, using secondary emission from SNRs, produced by accelerated CR.

It is worthwhile to separate the propagation effects on the spectrum from the acceleration in the source. Only the latter has a direct bearing on the DSA. At the same time, the CR propagation models have long been used to offset for the DSA deviations from the local spectra. A significant range in $\Delta q_{\text {prop }}=0.3-0.6$, depending on the particle scattering regime in the interstellar medium (ISM) (see, e.g., [8]), allowed one to link the local spectrum, $E^{-2.7}-E^{-2.8}$ to that of the DSApredicted, $E^{-2}-E^{-2.2}$. Being marginal in the first place, this link became more questionable after tighter constraints have been imposed on both the $\Delta q_{\text {prop }}$ (through independent measurements of the secondary to primary CR ratios, [9]) and the SNR source spectra (through secondary, primarily $\gamma$-emission generated by accelerated CRs through the $\pi^{0}$ channel, e.g., [10]). Even more persuasive indication of a steep source spectrum is the recent high-precision spectral index measured to be in the range $q=2.87 \pm 0.06$ below $500 \mathrm{GeV}$, by the CALET team [11], fully confirming the previously published AMS-02 proton spectrum [4]. These findings have added to an existing concern about the DSA capacity to account for the CR production in SNRs, see e.g. [12]. The old paradigm explaining steep spectra by imposing an exponential cut-off on the DSA- produced power-law does not comply with the new data, even if one assumes that the steep part of the spectrum originates from a nearby source with a cut-off in the sub-TeV range.

To address the problem of steep spectra, different scenarios have been recently proposed [13, 14]. The authors of [13] show that the steepened spectrum may be caused by the loss of CR energy due to turbulent magnetic field amplification during $\mathrm{CR}$ acceleration when the shock velocity is high. The scenario considered in [14] utilizes a well-known property of collisionless shocks to suppress the proton injection when the magnetic field ahead of the shock makes a large angle to its normal, typically $\theta_{\mathrm{Bn}} \gtrsim \pi / 4$. Two different spectrum steepening mechanisms can be associated with the variable magnetic field inclination. The first one is best represented in bilateral SNRs (a 
clear-cut example is SN 1006), where two regions of active particle acceleration $\left(\theta_{\mathrm{Bn}} \lesssim \pi / 4\right.$, polar caps) are separated by a region of oblique shock geometry (equatorial $\pi / 4 \lesssim \theta_{\mathrm{Bn}}<\pi / 2$ ). The steepening derives from the growth of the active acceleration zone in time, as the SNR expands. Compared to the case of a fixed acceleration area, freshly injected particles with lower energies are added to the growing acceleration zone at an area-integrated rate that increases with time. This enhanced production of low-energy particles naturally results in a steeper overall spectrum [14].The second steepening mechanism is associated with particle diffusion from the active acceleration zone into the neighboring oblique shock zone. As there is almost no particle confining turbulence there, these particles have a good chance to escape the accelerator. Since the diffusion coefficient typically grows with energy, a steeper spectrum is expected. This mechanism is much harder to quantify analytically since several competing factors are at play. First, when a particle reaches the interface between the active and inactive acceleration zones, the cross-field diffusion decreases, whereas the parallel diffusion, on the contrary, increases. This happens because the CR-driven turbulence level should decrease at the interface, while the parallel and perpendicular particle diffusivities are related as $\kappa_{\|} \kappa_{\perp} \simeq \kappa_{B}^{2}$. Here $\kappa_{B} \propto r_{g}$ is the Bohm diffusion that linearly growth with the particle gyro-radius $r_{g}$. So, the turbulence decrease will thus slow down the sideway losses but, at the same time, particles reaching the interface are more likely to escape along the field. If we add the wave self-generation by particles diffusing into the "quiet" zone of suppressed particle acceleration, the picture becomes even more complicated for analytic treatment. It may be noted that the second mechanism, by contrast with the first one, does not require the growth of acceleration area to produce steepening. Indeed, it results from particle losses incurred at the energy-dependent rate. This suggests that it can also work on a "patchy" shock where the regions of active and inactive acceleration are interspersed and coexist, not necessarily growing or shrinking. Therefore, it should apply to planar shocks and, to low-energy particles in spherical shocks where the shock sphericity is unimportant. This is the situation we study in this paper using hybrid simulations with a magnetic field changing periodically along the shock front.

It is important to note that the magnetic field ahead of the shock can vary at sufficiently large scales due to the cyclotron instability of high energy particles, escaping or diffusing further ahead of the shock. This would mean that the variable field component does not have to be preexisting in the ISM. Under these circumstances, the shock geometry becomes variable at scales significantly smaller than the shock radius but still larger than the gyroradii of the low-energy accelerated particles. The tilted magnetic field affects the injection of non-relativistic particles. This makes hybrid simulations suitable for studying the spectrum steepening mechanism outlined above, as no broad energy range and, respectively, large simulation box is required.

\section{Method and Simulation Set-up}

Hybrid simulations have been proven to be a valuable tool for investigating acceleration of ions at collisionless shocks. They have been applied to model shocks in the solar system [15, 16] as well as CR injection and acceleration at SNR shocks [17, 18]. In these simulations the ions, which determine the relevant scales, are treated kinetically, while the electrons are considered as a massless, charge neutralizing fluid, [19, and references therein]. In this paper we use a model, in which the electron pressure $p_{e}$ and the resistivity are both assumed to be isotropic. The pressure 
$p_{e}$ is assumed to follow an adiabatic equation of state with the adiabatic index $\gamma_{e}=5 / 3$. The fluid equations and the ion equations of motion are non-relativistic, as $|\vec{v}| \ll c$ holds during the injection phase. More details on our hybrid code can be found in [7].

In the simulations times are given in units of the inverse proton cyclotron frequency, $\omega_{c}=$ $e B_{0} / m_{p} c$, where $e, m_{p}$ are the protons charge and mass, respectively, $c$ denotes the velocity of light, and $B_{0}$ the amplitude of the background magnetic field. Distances are measured in terms of the proton skin depth $c / \omega_{p}$, with $\omega_{p}=\sqrt{4 \pi n_{0} e^{2} / m_{p}}$ being the proton plasma frequency, where $n_{0}$ is the far upstream density. The velocity is given in terms of the Alfvén velocity $v_{\mathrm{A}}=B_{0} / \sqrt{4 \pi n_{0} m_{p}}$. Spatially the motion of the ions is reduced to two dimensions, but all three components of the velocity and fields are included.

Since the modeling of an expanding SNR is not feasible by means of hybrid simulations, we focus on the influence of changing shock obliquity. In our simulation we mimic the variation of $\theta_{\mathrm{Bn}}$ over the shock surface in a $2 \mathrm{D}$ model set-up. As the shock is propagating in the $x$-direction, we vary $\theta_{\mathrm{Bn}}=\theta_{\mathrm{Bn}}(y)$ as function of the transverse coordinate by properly choosing $B_{x}$ and $B_{z}$ as functions of $y$, ensuring $\nabla \vec{B}=0$ and $|\vec{B}(y)|=B_{0}=$ const, Fig. 1 . The simulation is initialized by sending a super-sonic and super-alfvénic hydrogen plasma against a reflecting wall, placed at $x=0$. A shock forms upon the interaction of the counter-
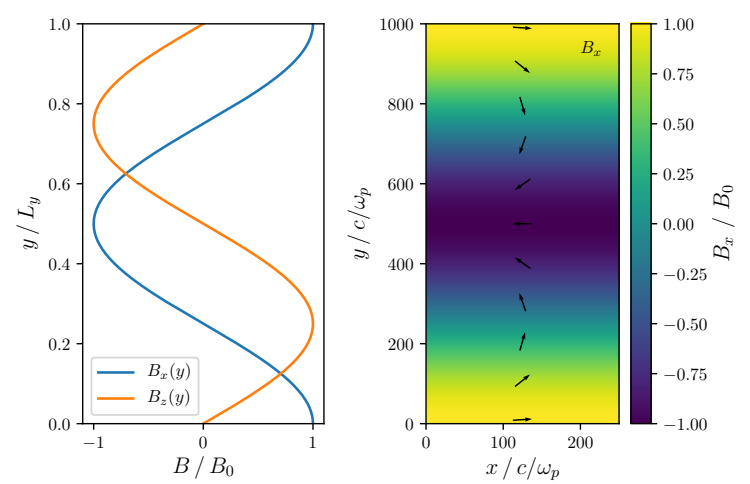

Figure 1: Background magnetic field $\vec{B}_{0}(y)$ distribution at $t=0$ for a setup with varying shock obliquity. (right) - initial dependence of the components $B_{x}$ and $B_{z}$ on the transverse coordinate (left) $-B_{x}(x, y)$, additionally $\theta_{\mathrm{Bn}}$ is depicted by the black arrows. propagating plasma streams and propagates in positive $x$-direction. Thus, the simulation frame is the downstream rest frame. Far upstream ions and electrons are assumed to be in thermal equilibrium with $\beta_{e}=\beta_{p}=1$. As the shock obliquity varies with the transverse coordinate, the transverse box size $L_{y}$ must be large enough, such that the results do not depend on $L_{y}$. For a similar set-up of

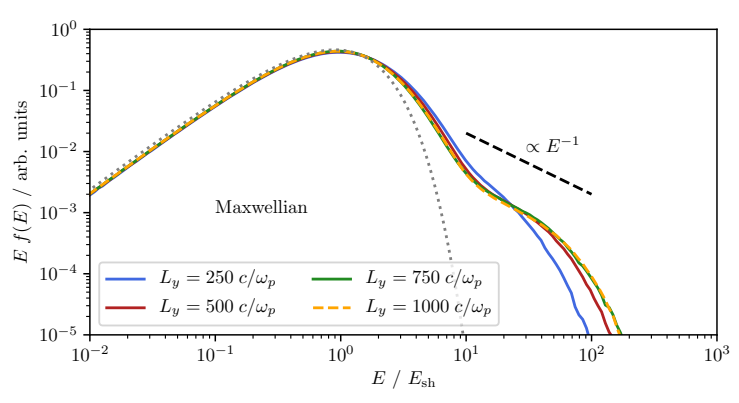

Figure 2: Downstream energy spectra for different transverse box sizes at $t=400 \omega_{c}^{-1}$.

stream flow velocity of $v_{0}=10 v_{\mathrm{A}}$. the magnetic field $\vec{B}_{0}=B_{0}\left(\sin \frac{\pi y}{L_{y}}, 0,\left|\cos \frac{\pi y}{L_{y}}\right|\right)$, we have performed simulations for different transverse box sizes and investigated the convergence of the downstream energy spectra, Fig. 2. For the two largest $L_{y}$ the resulting energy spectra are almost identical. Hence we are confident that a transverse size of $L_{y}=1000 \mathrm{c} / \omega_{p}$ yields converged results. The length of the simulation box is set to be $L_{x}=8000 c / \omega_{p}$ with a cell size of $\Delta_{x}=\Delta_{y}=$ $0.5 c / \omega_{p}$. We use 16 particles per cell and a time step of $\Delta t=0.001 \omega_{c}^{-1}$ for an initial up- 


\section{Simulation Results}

When a shock propagates into an environment, in which the inclination of the magnetic field to the shock front varies, different regions can be distinguished, depending on whether the background magnetic field is rather parallel or perpendicular to the shock normal. Figure 3 shows a snapshot of the absolute value of the magnetic field $|\vec{B}(x, y)|$ in the simulation domain at $t=300 \omega_{c}^{-1}$. The shock transition at $x \sim 1200 c / \omega_{p}$, the corresponding increase in density and the compression of the magnetic field are clearly visible. The three regions, indicated by the red rectangles on Fig. 3a) correspond to the areas where the shock is perpendicular (1), quasi-parallel (2) or parallel (3). The lower panels (Fig. 3b-d) show the components of the magnetic field averaged over $y$ in these regions. The proton injection is only efficient at quasi-parallel shocks. This can be obtained from simple kinematic considerations [20] and has been observed in Monte-Carlo [21] and hybrid simulations [18]. The behavior of the fields upstream of the shock transition reflects this fact. Only in regions where the angle between the upstream magnetic field and the shock normal is small $\left(\theta_{\mathrm{cr}}=\pi / 4\right)$ ions can return upstream and excite waves (frames (b) and (c)). In the region, where the shock has a quasi-perpendicular geometry (Fig. 3d) no waves are present in the upstream.

The spatial distribution of protons with positive longitudinal velocity $v_{x}>0$, is shown in

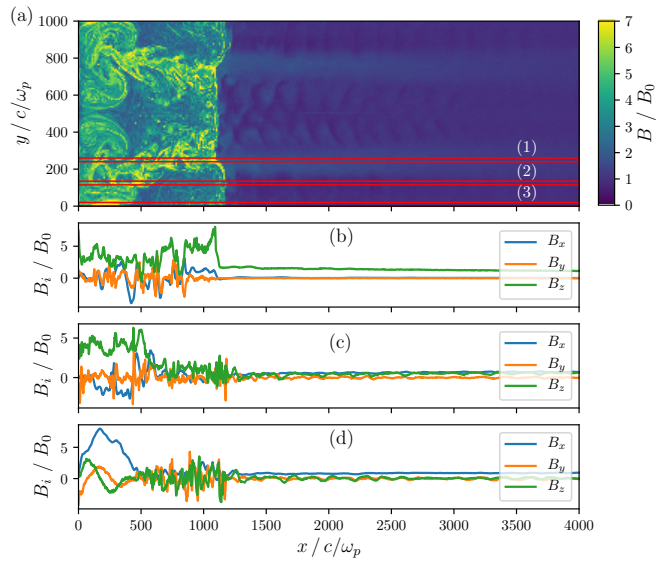

Figure 3: (a) - Magnetic field amplitude $|B|$ at $t=300 \omega_{c}^{-1}$. (b)-(d) - Components of the magnetic field in regions of different shock obliquity, indicated in red on panel (a). Fig. 4 in the vicinity of the shock. Upstream and downstream regions are noticeably different. Many particles with positive $v_{x}$ exist in the downstream due to the turbulent fields there (note, that the simulation frame is the downstream rest frame). On the contrary, only a relatively low amount of particles have positive $v_{x}$ in the upstream. These particles are either directly reflected from the shock or have returned from the downstream plasma and are mainly present in regions of $\theta_{\mathrm{Bn}} \leq 45^{\circ}$,



Figure 4: Density of protons with $v_{x}>0$ for (a) - the model set-up with variable obliquity and (b) - a simulation of a quasi-parallel shock, both at $t=300 \omega_{c}^{-1}$.
Fig. 4a). For comparison the spatial distribution of protons having positive longitudinal velocity $v_{x}>0$ in the vicinity of the shock, is also shown for a simulation with constant obliquity $\theta_{\mathrm{Bn}}=20^{\circ}$ in Fig. 4b). Here, the number of particles with $v_{x}>0$ upstream of the shock is almost independent of the transverse coordinate and variations are only present close to the shock transition.

In order to investigate the spectral steepening we have obtained energy spectra from the particle data from the simulation using a logarithmic binning. In Figure 5 we compare the proton down- 
stream energy spectrum from our model set-up (solid red line) to the spectrum from a simulation of a quasi-parallel shock with $\theta_{\mathrm{Bn}}=20^{\circ}$ (solid blue line). In both cases the spectra are obtained at $t=400 \omega_{c}^{-1}$ and are integrated through the whole downstream region. The energy is given in terms of $E_{\mathrm{sh}}=m_{p} v_{0}^{2} / 2$. The obvious difference between these spectra is the normalization of the power-law tail, which is larger for the quasi-parallel shock, since the injection efficiency is high over the whole shock surface. In Figure 5 we also show the energy spectra in different regions at an earlier time, $t=100 \omega_{c}^{-1}$, as a spatial dependence of the spectra can be expected (see for example [22], where spatially dependent momentum distribution functions have been observed in simulations of collisionless shocks containing large-scale magnetic-field variations). At early times the spectrum in the quasi-parallel region is similar to the spectrum obtained in a simulation of a fully quasi-parallel shock. This is because the injection efficiency does not depend largely on $\theta_{\mathrm{Bn}}$, as long as the shock is quasi-parallel [18]. The spectrum obtained in the quasi-perpendicular region shows the presence of ions which are mildly energized by shock drift acceleration [23] while the formation of a power-law tail is suppressed. But in contrast to fully (quasi-)perpendicular shocks a bump is visible in the spectrum, which can be attributed to accelerated ions, which have diffused from the quasi-parallel region into the quasi-perpendicular one. This effect leads to an alignment of the spectra in both regions over time.

The energy spectra, shown in Fig. 5 clearly indicate that the power-law exponent differs for the simulations with constant or variably field obliquity. To make it more evident, we have calculated the power-law index

$$
q=-\frac{\mathrm{d} \ln (f(E))}{\mathrm{d} \ln E}
$$

from the computed spectra as function of energy and plot the result in the lower panel of Fig. 5. Here the blue line corresponds to a set-up with $\theta_{\mathrm{Bn}}=20^{\circ}=$ const, while the red line corresponds to a simulation with variable obliquity. Common to both spectra is the beginning of the power-law behavior around $E=10 E_{\mathrm{sh}}$. At high energies the spectra exhibit an exponential cut-off due to the limited simulation time and box size. In the energy region $15<E / E_{\mathrm{sh}}<40$ the computed index $q^{\prime}=-\ln f(E) / \ln E$ does not change significantly, with the mean value being larger for the simulation set-up with vari-
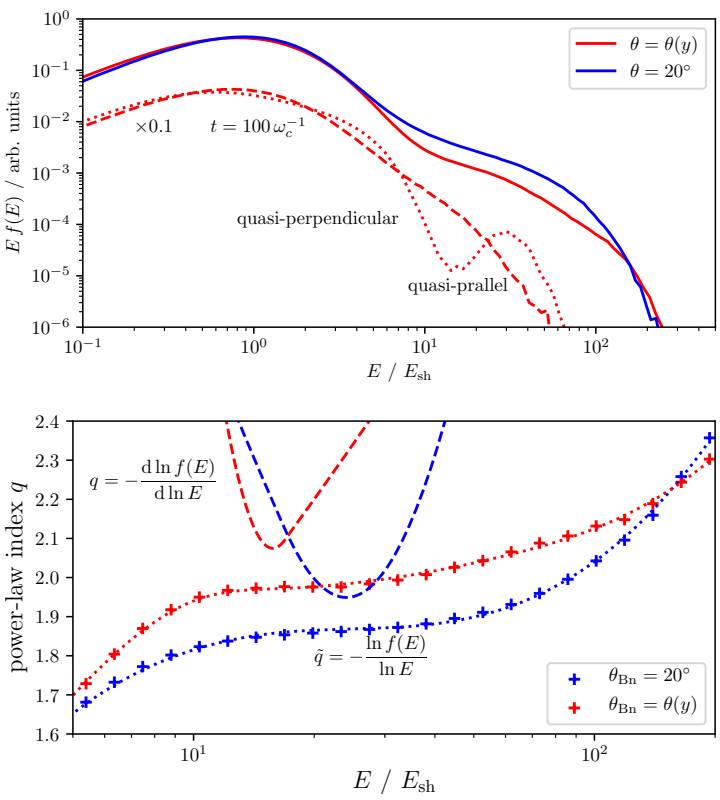

Figure 5: (top) Downstream energy spectra: a simulation of a quasi-parallel shock (blue) and our model with variable shock obliquity (red). (bottom) Powerlaw spectral index calculated from the downstream energy spectra at $t=400 \omega_{c}^{-1}$ : blue line $-\theta_{\mathrm{Bn}}=20^{\circ}=$ const, red line - variable obliquity.

able shock obliquity (red line). The difference of the spectral indices (Eq. 3.1) obtained from the simulations is $\Delta q=q_{\mathrm{var}}-q_{\mathrm{const}} \approx 0.1-0.15$. 


\section{Summary}

In this work we have investigated the influence of a variation of the collisionless shock obliquity over the shock surface on the acceleration of protons by means of hybrid modeling. Limited in resources, our simulations have captured a new physical phenomenon in the DSA - the spectrum steepening associated with the variation of shock obliquity along its face. We find that for regions of parallel and perpendicular shock propagation coexisting in one simulation box, the DSA process starts and proceeds at high efficiency in the quasi-parallel region. However, while the particle energy increases, some of them diffuse to the quasi-perpendicular shock domain. The resulting shock-generated downstream spectrum is noticeably steeper than canonical DSA result obtained for a quasi-parallel shock. We observe no strong shock deceleration in the quasi-perpendicular field direction compared to the quasi-parallel one. It is worth noting, that while the spectrum steepening found in our simulations may be regarded as relatively small, $\Delta q=0.1-0.15$, it is significant for a number of reasons. First, it is likely to be scalable to larger boxes and longer simulation time, so that somewhat larger $\Delta q$ values can be expected for realistic SNR conditions. Second, the mechanism operates at relatively low energies while at higher energies a different but closely related mechanism, described in the Introduction, should take over. Again, a steeper overall spectrum in the SNRs will then result, as suggested by observations. Finally, we emphasize, that the highly determined DSA predictions combined with extremely accurate CR data have proved even small variations of the spectral index to be meaningful and even testable.

\section{Acknowledgments}

The research was supported by DFG grant TL 2479/2-1 and by NASA ATP-program within grant 80NSSC17K0255. The authors acknowledge the North-German Supercomputing Alliance (HLRN) for providing the computational resources for the simulations.

\section{References}

[1] K. M. Schure, A. R. Bell, L. O'C Drury, and A. M. Bykov, "Diffusive shock acceleration and magnetic field amplification,” Space Science Reviews, vol. 173, pp. 491-519, Nov 2012.

[2] H. S. Ahn et al., "The energy spectra of protons and helium measured with the ATIC experiment," Advances in Space Research, vol. 37, pp. 1950-1954, 2006.

[3] O. Adriani et al., "Pamela measurements of cosmic-ray proton and helium spectra," Science, vol. 332, no. 6025, pp. 69-72, 2011.

[4] M. Aguilar et al., "Precision measurement of the helium flux in primary cosmic rays of rigidities 1.9 gv to $3 \mathrm{tv}$ with the alpha magnetic spectrometer on the international space station," Phys. Rev. Lett., vol. 115, p. 211101, Nov 2015.

[5] P. Serpico, "Possible physics scenarios behind cosmic-ray anomalies," in 34th International Cosmic Ray Conference (ICRC2015), vol. 34, p. 9, Jul 2015.

[6] M. Malkov, "Newly-discovered anomalies in galactic cosmic rays: Time for exotic scenarios?," Nuclear and Particle Physics Proceedings, vol. 297-299, pp. 20 - 30, 2018. Cosmic Ray Origin Beyond the Standard Models. 
[7] A. Hanusch, T. V. Liseykina, and M. Malkov, "Acceleration of cosmic rays in supernova shocks: Elemental selectivity of the injection mechanism," The Astrophysical Journal, vol. 872, p. 108, feb 2019.

[8] A. W. Strong, I. V. Moskalenko, and V. S. Ptuskin, "Cosmic-ray propagation and interactions in the galaxy," Annual Review of Nuclear and Particle Science, vol. 57, no. 1, pp. 285-327, 2007.

[9] M. Aguilar et al., "Precision measurement of the boron to carbon flux ratio in cosmic rays from $1.9 \mathrm{gv}$ to $2.6 \mathrm{tv}$ with the alpha magnetic spectrometer on the international space station," Phys. Rev. Lett., vol. 117, p. 231102, Nov 2016.

[10] F. Aharonian, R. Yang, and E. de Oña Wilhelmi, "Massive stars as major factories of Galactic cosmic rays," Nature Astronomy, vol. 3, pp. 561-567, Mar. 2019.

[11] O. Adriani, Y. Akaike, K. Asano, Y. Asaoka, M. G. Bagliesi, E. Berti, G. Bigongiari, W. R. Binns, S. Bonechi, and M. Bongi, "Direct measurement of the cosmic-ray proton spectrum from 50 gev to 10 tev with the calorimetric electron telescope on the international space station," Phys. Rev. Lett., vol. 122, p. 181102, May 2019.

[12] S. Gabici, C. Evoli, D. Gaggero, P. Lipari, P. Mertsch, E. Orlando, A. Strong, and A. Vittino, "The origin of Galactic cosmic rays: challenges to the standard paradigm," arXiv e-prints, p. arXiv:1903.11584, Mar 2019.

[13] A. Bell, J. Matthews, and K. Blundell, "Cosmic ray acceleration by shocks: spectral steepening due to turbulent magnetic field amplification,” arXiv e-prints, p. arXiv:1906.12240, Jun 2019.

[14] M. Malkov and F. Aharonian, "Cosmic Ray Spectra in Supernova Remnants - I. Loss-Free Self-Similar Solution,” arXiv e-prints, p. arXiv:1901.01284, Jan 2019.

[15] Y. Lin and X. Y. Wang, "Three-dimensional global hybrid simulation of dayside dynamics associated with the quasi-parallel bow shock," Journal of Geophysical Research: Space Physics, vol. 110, no. A12, 2005.

[16] J. Giacalone, "The acceleration of thermal ions at a strong, quasi-parallel interplanetary shock: A hybrid simulation,” Journal of Physics: Conference Series, vol. 900, p. 012008, sep 2017.

[17] K. B. Quest, “Theory and simulation of collisionless parallel shocks,” Journal of Geophysical Research: Space Physics, vol. 93, no. A9, pp. 9649-9680, 1988.

[18] D. Caprioli and A. Spitkovsky, "Simulations of ion acceleration at non-relativistic shocks. i. acceleration efficiency," The Astrophysical Journal, vol. 783, p. 91, feb 2014.

[19] A. S. Lipatov, The hybrid multiscale simulation technology: an introduction with application to astrophysical and laboratory plasmas. Springer Science \& Business Media, 2013.

[20] M. A. Malkov and H. J. Völk, "Theory of ion injection at shocks.," Astronomy and Astrophys., vol. 300, p. 605, Aug. 1995.

[21] D. C. Ellison, F. C. Jones, and M. G. Baring, "Acceleration rates and injection efficiencies in oblique shocks,” Astrophys. J., vol. 453, no. astro-ph/9506076, pp. 873-882, 1995.

[22] F. Guo, J. R. Jokipii, and J. Kota, "Particle acceleration by collisionless shocks containing large-scale magnetic-field variations," The Astrophysical Journal, vol. 725, pp. 128-133, nov 2010.

[23] J. Park, J. C. Workman, E. G. Blackman, C. Ren, and R. Siller, "Particle-in-cell simulations of particle energization from low mach number fast mode shocks," Physics of Plasmas, vol. 19, no. 6, p. 062904, 2012. 